\title{
BMJ Open Childhood abuse and its association with adults' mental health problems: a cross-sectional study among men and women in the Yangon Region of Myanmar
}

\author{
Win Thuzar Aye (D) , ${ }^{1,2}$ Lars Lien, ${ }^{3}$ Hein Stigum, ${ }^{4}$ Espen Bjertness ${ }^{4}$
}

To cite: Aye WT, Lien L, Stigum H, et al. Childhood abuse and its association with adults' mental health problems: a cross-sectional study among men and women in the Yangon Region of Myanmar. BMJ Open 2021;11:e045870. doi:10.1136/ bmjopen-2020-045870

- Prepublication history for this paper is available online. To view these files, please visit the journal online (http://dx.doi. org/10.1136/bmjopen-2020045870).

Received 15 October 2020 Accepted 25 0ctober 2021

Check for updates

(C) Author(s) (or their employer(s)) 2021. Re-use permitted under CC BY-NC. No commercial re-use. See rights and permissions. Published by BMJ.

For numbered affiliations see end of article.

Correspondence to Dr Win Thuzar Aye; winthuzaraye@gmail.com

\section{ABSTRACT}

Objectives To estimate the prevalence of emotional, physical and sexual childhood abuse, and symptoms of post-traumatic stress disorder (PTSD) and to examine the association between childhood abuse and adult mental health problems, including mental distress and PTSD symptoms.

Design A community-based cross-sectional study was conducted. Childhood abuse was assessed with the NorVold Abuse Questionnaire, and mental distress and symptoms of PTSD were measured using the Hopkins Symptom Checklist 10 and the Impact of Event Scale-Revised, respectively. The Wald test and multiple linear regression analysis were applied for testing differences between proportions and the association between childhood abuse and adult mental health outcomes, respectively.

Setting Urban and rural areas of the Yangon Region, Myanmar.

Participants A total of 2377 men and women aged 18-49 years were included. Institutionalised people, monks, nuns and individuals deemed too ill physically and/or mentally to participate were excluded.

Results Overall, $21.1 \%$ (95\% $\mathrm{Cl} 18.8$ to 23.6 ) reported any form of childhood abuse, $10.4 \%$ ( $95 \% \mathrm{Cl} 8.9$ to 12.4$)$ physical abuse, $10.4 \%$ (95\% Cl 8.8 to 12.2) emotional abuse and $7.3 \%$ (95\% Cl 5.7 to 9.3) sexual abuse. Childhood abuse was more common in women (29.8\%) than in men (12.4\%). The prevalence of PTSD symptoms in the total sample was $6.6 \%$. After adjusting for confounding variables, positive associations were found between childhood abuse with adult mental distress and PTSD symptoms among women and older men. Conclusions Childhood abuse is prevalent among both men and women in the Yangon Region of Myanmar and associated with adult mental health problems. Approximately $7 \%$ of people report PTSD symptoms. It should prompt local health professionals and policy makers to establish prevention programmes to eliminate violence against children and to organise services for victims of childhood abuse. Care should be taken in generalising findings for less populated areas.

\section{INTRODUCTION}

Childhood abuse has severe impacts on the victim's social life and physical and mental health, and it is a violation of human rights. ${ }^{12}$ The WHO has defined child abuse or child
Strengths and limitations of this study

- This is the first population-based study conducted in Myanmar estimating the prevalence of childhood abuse and post-traumatic stress disorder symptoms and the association between childhood abuse and adult mental health outcomes in both sexes.

- Its high response rate and use of well-established measures of data and an analysis strategy based on a directed acyclic graph strengthen the external and internal validity of the study.

- Under-reporting might have resulted due to the sensitive nature of questions about childhood abuse and also mental health.

- Recall bias could be present due to the retrospective nature of the questions.

- Its cross-sectional design means that it is not possible to infer a causal relationship between childhood abuse and adult mental health outcomes.

maltreatment as 'all forms of physical and/or emotional ill-treatment, sexual abuse, neglect or negligent treatment or commercial or other exploitation, resulting in actual or potential harm to the child's health, survival, development or dignity in the context of a relationship of responsibility, trust or power'. ${ }^{2}$ In this study, the term 'childhood abuse' refers to physical, sexual, and emotional or psychological abuse perpetrated on persons under 18 years of age by parents, caregivers, peers or strangers.

The prevalence of childhood abuse varies across countries and regions as well as by gender and age. Global estimates of the prevalence of past-year violence among girls and boys aged 2-17years old from 96 countries between 2014 and 2015 found that 1.7 billion children worldwide were exposed to interpersonal violence in their daily lives, ${ }^{3}$ and over $54 \%$ of children experienced either physical, 
sexual, emotional bullying or witnessed violence during the past year. ${ }^{3}$ The highest prevalence of violence against children was found in Asia (64\%) and the lowest was in Europe (12\%). ${ }^{3}$ The WHO's 2014 global status report on violence prevention stated that childhood abuse rates of $36 \%$ psychological, $23 \%$ physical and $18 \%$ sexual abuse were perpetrated against girls, and $8 \%$ sexual abuse was perpetrated against boys during childhood. ${ }^{2}$ Findings from the UN Multi-country Study on Men Violence in Asia and the Pacific Region showed that the prevalence of individuals who experienced childhood trauma, including emotional abuse and neglect, physical abuse and sexual abuse, as well as witnessing abuse of their mother, varied from $59 \%$ to $92 \%$ in men and from $44 \%$ to $84 \%$ in women. ${ }^{4}$

According to a 2016 Global School-Based Student Health Survey (GSHS) of 13-17 year-old male and female students in Myanmar, 32.7\% of students reported having been physically abused during the past 12 months. Among these, $39.8 \%$ were boys and $26.3 \%$ were girls. ${ }^{5}$ Currently, child abuse is a hidden epidemic and a public health emergency in Myanmar. ${ }^{6}$ The rate of child abuse, especially sexual abuse, threatens the society. ${ }^{6} 7$ Myanmar's vulnerability due to conflicts, natural disasters, poverty, and its social norms and traditional attitudes may further exacerbate its child abuse problem. ${ }^{8}$ Because of poverty, some girls work as domestic servants, which puts them at risk of sexual assault by their employer, ${ }^{9}$ and many victims choose not to report these attacks for various reasons, including a significant power discrepancy between the perpetrator and the child and fear of consequences of reporting the abuse, including stigma and retaliation. Furthermore, many parents in Myanmar seem to be unaware of the risk of child abuse, ${ }^{9}$ and because of poverty, some parents in rural areas cannot afford to send their children to government schools. As an alternative, they are sent to the monastic school, which offers free or low-cost education and accommodation. Nevertheless, monastic life has become a traumatising experience marked by physical and/or sexual abuse for some children. ${ }^{7}$ Compounding these societal problems, there is still a lack of services for victims of violence in terms of social and mental support. ${ }^{9}$ Although the Ministry of Social Welfare, Relief and Resettlement has redrafted the 1993 Child Law, the child sexual abuse context is not adequately addressed in the legislation. ${ }^{9}$

PTSD may occur after traumatic events including the threat of death or serious injury due to violence or a disaster, the unexpected death of a loved one or accidents. ${ }^{10}$ Prevalence estimates of PTSD in the general population in low-income and middle-income countries (LMICs) are scarce ${ }^{11} 12$ since most studies are conducted in relation to high-risk populations ${ }^{13}$ such as refugees ${ }^{1314}$ and survivors after disaster. ${ }^{15}$ For example, recent studies conducted among refugees from Myanmar living in Australia, ${ }^{13}$ refugees from Myanmar in Buffalo, New York in the USA ${ }^{14}$ and survivors after disaster in 2008,
Myanmar, ${ }^{15}$ reported a prevalence of PTSD at 27\%, 50.4\% and $20 \%$ respectively.

The recent World Mental Health Survey analysed data collected on men and women ages 18+ years from population-based studies between 2001 and 2012. The data represented the general population of 4 low to lowermiddle-income countries (LLMIC), 6 upper-middleincome countries (UMICs) and 13 high-income countries (HICs). ${ }^{11}$ The survey reported 29 types of traumatic exposures and estimated that the lifetime prevalence of PTSD was $3.9 \%$ among the study population and $5.6 \%$ among the the trauma-exposed population. ${ }^{11}$ The lifetime prevalence of traumatic exposures was $69.1 \%$ in LLMICs, $63.2 \%$ in UMICs and $72.4 \%$ in HICs, respectively, and the corresponding prevalence of PTSD among those exposed was $3.0 \%, 3.6 \%$ and $6.9 \%$, respectively. ${ }^{11}$

Violence against children is an important risk factor for mental health problems, and a majority of previous studies on this topic have documented that childhood abuse survivors suffer mental health problems in adulthood. ${ }^{16-19}$ A systematic review and meta-analysis of 111 review articles published between 1988 and $2017^{17}$ found that child maltreatment was associated with a wide range of mental health problems in adulthood, including PTSD, anxiety disorders, depression, bipolar disorder, psychosomatic disorders and personality disorders. A populationbased study on health among the Sami, an indigenous population group, and the non-Sami population in Norway showed that childhood abuse was positively associated with adult mental distress and post-traumatic stress symptoms. $^{18}$

To 'end abuse, exploitation, trafficking and all forms of violence against and torture of children' is a priority target of the Sustainable Development Goals (SDGs). ${ }^{19}$ Research on the extent of the problem and the consequences of childhood violence is well documented in HICs. However, there is still an information gap in LMICs. $^{2}{ }^{3} 19$ Until now, prevalence estimates of child abuse in Myanmar have been drawn from one schoolbased study that included 2838 interviews with students aged 13-17 years from 50 schools. ${ }^{5}$ Some data are gathered from conflict areas and address only women and sexual assault. ${ }^{9}$ No comprehensive data are available that address the prevalence of different forms of abuse in childhood and their association with mental health problems in adulthood in Myanmar. The objectives of this study conducted with men and women aged 18-49years old in the Yangon Region of Myanmar were to estimate the prevalence of childhood abuse (psychological or emotional, physical and sexual) and PTSD symptoms and to examine the association between childhood abuse with adult mental distress and PTSD symptoms across gender.

\section{METHODS}

\section{Study design and setting}

A household-based cross-sectional survey was conducted in the Northern and Southern Districts of the Yangon 
Region of Myanmar between October and November 2016. The Yangon Region is the most developed and densely populated area of Myanmar. It comprises four districts (Eastern, Western, Northern and Southern); only two districts (Northern and Southern) have both urban and rural areas and were, therefore, purposively selected. A representative sample of men and women ages 18-49 years was included from these two districts. Individuals who were deemed physically and/or mentally too ill to participate, in addition to monks, nuns, soldiers and institutionalised people, were excluded. The samplesize calculation was based on the population distribution in the Northern District $(64.8 \%)$ and the Southern District $(35.2 \%)^{20}$ and the expected prevalence of exposure to domestic violence perpetrated against women by their spouses $(21 \%) .{ }^{21}$ We included a sample size of 2400 men and women to reach levels of statistical power of $80 \%$ and to account for a $20 \%$ non-responses rate in order to meet the objectives of the study. Respondents were selected using a multistage sampling method. Initially, two of the four districts of the Yangon Region were purposively selected. We generated a list of urban wards (an urban subunit of a township) and rural villages in each district; there are 125 wards and 235 villages in the Northern District and 110 wards and 375 villages in the Southern District. From the two districts, 8 wards and 8 villages were randomly selected for a total of 16 wards and 16 villages included in the study. In these wards and villages, the households were randomly selected based on the proportion of the population in urban (wards) and rural (villages) areas. According to the lists of households obtained from the local authorities, we randomly selected 2950 households: 336 and 1034 households from urban wards of the Southern and Northern Districts, respectively, and 686 and 894 households from rural villages in the Southern and Northern Districts, respectively. In 89 households, it was not possible to contact household members. From the remaining 2861 households, we invited only one respondent per household to participate in the study. Among the invitees, 130 declined to participate, for a response rate of $92.6 \%$ (2731 of 2950). In each household, one person was asked to list the family members within the age range of 18-49 years, and then one participant was randomly selected using the sealedenvelope method. ${ }^{22}$ One woman and one man were randomly selected from every other household. During data collection, it was not possible to ensure privacy for 152 of the participants, and therefore, these interviews were terminated. An additional 202 participants had incomplete questionnaires and, thus, were excluded from the analyses. The final sample included 2377 participants comprising 1190 men and 1187 women.

\section{Data collection and measurement}

The data collection was carried out by the principal researcher and 12 well-trained male and female interviewers. A 2-day training session was provided to ensure consistency with regard to interview techniques, the sampling procedure, the objectives of the study and ethical issues; to build trust between interviewers and respondents; and to ensure the confidentiality and safety of both the interviewers and the respondents. We followed the WHO ethics and safety guidelines for research about violence against women, which has been translated to Myanmar's Burmese language. ${ }^{23}$ The Norvold Abuse Questionnaire (NorAQ) for childhood violence ${ }^{24}$ was translated to the Burmese language by an expert from the gender-based violence organisation (United Nations Population Fund) in Myanmar. Additionally, the Impact of Event Scale-Revised (IES-R) questionnaire for PTSD symptoms $^{25}$ and the Hopkins Symptom Checklist 10 $\left(\right.$ HSCL-10) ${ }^{26}$ for mental distress were translated to Burmese by a psychiatrist. All questionnaires were backtranslated to English by another person with Englishlanguage proficiency in order to ensure consistency and accuracy.

We conducted a pilot survey in order to refine the survey instruments. All interviews were conducted face-to-face by trained, sex-matched field workers using questionnaires. Interviews took place either in the home or outdoors in private areas. All respondents provided written informed consent. We informed participants that the questions could be sensitive, and that they had the right to withdraw from the study at any time or refrain from answering particular questions. If the invited person from the household was not at home or refused to participate, this was regarded as a non-response. The completeness of the questionnaires was further checked by the principal investigator on a daily basis. Missing answers were completed with a revisit to the household on the same day.

\section{Patient and public involvement}

There was no involvement of patients or the public in designing, recruiting and conducting the study.

\section{Study variables}

The childhood abuse questionnaire was adopted from the NorAQ. ${ }^{24}$ The validity of the Swedish version for both adult men and women showed that the instrument has good reliability and validity. ${ }^{24}$ In addition, the Arabic version of the NorAQ conducted in Jordan demonstrated a Cronbach's $\alpha$ of $0.75,{ }^{28}$ which is in agreement with our study (Cronbach's $\alpha=0.73$ ). The NorAQ included three dimensions: emotional, physical and sexual abuses. Respondents who gave a positive response to any of the emotional abuse questions, for example, 'Have you experienced that someone (1) systematically tried to repress or humiliate you, (2) threatened to or took control of your contacts with others, or (3) that you lived in fear because of threats made against you?' were noted as having experienced emotional abuse. Similarly, respondents who gave a positive response to any of the physical abuse questions, for example, 'Have you experienced that someone has (1) hit, punched or held you against your will by (2) hitting you with their fist(s) or a hard object and/ or kicking, pushing and beating you or (3) threatened 
you with a weapons?' were considered as having been subjected to physical abuse. Similarly, a positive response to any of the sexual abuse questions, for example, 'Have you experienced that someone has (1) touched parts of your body against your will, (2) sexually humiliated you, (3) touched your genitalia against your will, (4) put his penis or other objects into your vagina, mouth or rectum against your will?' was considered as having experienced sexual abuse. Furthermore, respondents who gave a positive response to any of the aforementioned questions were asked whether the abuse happened when they were children $(<18$ years old), as an adult $(\geq 18$ years old) or during both their childhood and adulthood. Any childhood abuse was defined as the respondent having experienced any type of emotional, physical and/or sexual abuse during childhood ( $<18$ years old). The individuals who answered 'yes' to at least one of the child abuse questions were considered as having suffered from childhood abuse. Similarly, individuals who responded yes to at least one of the adult abuse questions were defined as having suffered from adult abuse.

\section{Outcome variables}

The revised 12-item version of the IES-R was used for assessing PTSD symptoms. It is one of the most commonly used questionnaires for measuring PTSD symptoms after exposure to one or more traumatic events ${ }^{25}$ and has been translated into a number of languages including Spanish, French, Chinese, Japanese, German ${ }^{29}$ and Myanmar. ${ }^{15}$ Several studies have shown that the IES-R has good reliability and validity in various populations and multiple cultures where trauma has been exposed. ${ }^{30-32}$ A study conducted in Taiwan and Myanmar showed that the internal consistency coefficients (Cronbach's alpha) of the IES-R using the Chinese and Myanmar version were $0.94^{31}$ and $0.98 .^{15}$ In the present study, the total IES-R score demonstrated good internal consistency $(\alpha=0.96)$.

The assessment of PTSD symptoms began with a question to identify participants who reported having been exposed to trauma during their lifetime: 'Have you ever experienced or witnessed or had to deal with an extremely traumatic events such as disaster, violence, war etc?'. Those who had been exposed to trauma were further asked about symptoms of PTSD during the previous 7 days. They were asked if (1) reminders of the trauma caused them to have a physical reaction, (2) whether they avoided becoming upset when they were reminded, (3) if they had tried to remove it from their memory, (4) whether they had waves of strong feelings about the trauma, (5) if they had dreams about it, (6) if they ever felt as though it had not really happened or it was not real, (7) if they tried not to talk about it, (8) whether pictures about it popped into their minds, (9) if they still had a lot of feelings about it but I did not deal with them, (10) if they tried not to think about it, (11) whether they had trouble falling asleep or staying asleep, and (12) if they stayed away from reminders of the trauma.
The response format in the IES-R referring to symptom severity included $0=$ not at all, $1=$ a little bit, $2=$ moderately, $3=$ quite $\mathrm{a}$ bit and $4=$ extremely. The scores of the 12 items of the IES-R range from 0 to 48 . The IES-R is not a diagnostic tool for PTSD; therefore, there is no specific cut-off score. The optimal cut -off point for a Myanmar version of 22 items of the (IES-R-M) was determined to be 33, which had both good sensitivity of $86 \%$ and specificity of $98 \%$ for the evaluation of PTSD symptoms among survivors after natural disaster. ${ }^{15}$ Besides, an abbreviated six-item version of the IES-R has demonstrated sensitivity $(55 \%-$ 96\%) and specificity (74\%-99\%) at a cut-off of 7-13 for PTSD symptoms in evaluations of survivors of trauma, natural disasters and personal violence in four Norwegian and Welsh samples. ${ }^{32}$ The cut-off of 10 in the previous study (six-item version) ${ }^{32}$ showed good overall efficiency (0.89), sensitivity $(0.80)$ and specificity $(0.92)$. Hence, the cut-off point above 20 for the 12-item version of IES-R was selected for presence of PTSD symptoms in the present study (ie, the cut-off is the double of the 6-item version).

Mental distress was assessed with the HSCL- $10,{ }^{26}$ which measures symptoms of anxiety and depression during the previous week. It consists of 10 items on a 4-point scale ranging from 1 (not at all) to 4 (extremely). The tool has high sensitivity and specificity to identify distressed and non-distressed respondents in the general population, and values above the cut-off of $1.85^{26}$ are significantly associated with an increased risk of mental distress. The reliability and validity of the HSCL-10 as a screening measure for mental distress has been demonstrated in community surveys in Norway ${ }^{26}$ and Pakistan. ${ }^{33}$ In the present study, the internal consistency (Cronbach's $\alpha$ : 0.85 ) was similar to the Pakistani population-based study $(0.86) .{ }^{33}$ In the multivariable analysis, mental distress was used as a continuous variable. ${ }^{26}$

\section{Sociodemographic variables}

Sociodemographic variables included age, sex, urban/ rural location, education and marital status. Ages (in years) of the respondents were categorised into age groups of 18-29, 30-39 and 40-49. For educational levels, we categorised the number of years at school into three separate groups: $0-5$ years, $6-11$ years and more than 11 years of schooling. Age and education (years of schooling) were used as continuous variables in the multivariable analysis. Regarding marital status, we categorised respondents as having or having already had a partner and never having had a partner.

Behavioural indicators of childhood violence included a partner's controlling behaviour, fear of a partner and witnessing parents beating each other. Partners' controlling behaviours included keeping him/her from seeing friends, restricting his/her contact with family, insisting on knowing where he/she is at all times, and becoming angry when he/she talks with other women or men. If at least one of the aforementioned actions were taken by the partner, the partner was referred to as a controlling husband or wife. 
For the different impairments (mobility, vision, hearing, personal hygiene/dressing, and memory or concentration), the responses were categorised as yes or 'no'. Muscle and skeletal pain were measured by the following questions: 'Have you in the last 12 months experienced pain several times in the head, neck/shoulders, arms/ legs/knees, stomach, and/or back?' with responses being yes or 'no' ${ }^{34}$ If a respondent answered yes to one or more of the body sites listed, it was considered as musculoskeletal pain. ${ }^{34}$ Self-perceived health status was based on the question: 'In general, how would you characterise your current health?' The response options were poor, not very good, good and very good. The variable was operationalised as 'poor health' (poor, not very good) and 'good health' (good, very good).

\section{Statistical methods}

Epidata Entry V.3.1 version was used for double-data entry and Stata/SE 16.0 was applied to perform all statistical analyses. We used the survey set command 'svy' due to the complex survey design and performed separate analyses for men and women. We estimated the prevalence of different types of childhood abuse, traumatic events and PTSD symptoms (IES-R score $\geq 20$ ), and we report the prevalence of any childhood abuse by sociodemographic, behaviour and health status variables. We present the prevalence of PTSD symptoms by sociodemographic variables, health status and different types of childhood abuse. Prevalence was estimated with a $95 \%$ CI and frequency measurements, and the Wald $\chi^{2}$ test was conducted to assess the differences between the two proportions. The non-normal distribution of PTSD symptoms was reported as median and $\mathrm{IQR}$, and the Wilcoxon signed-rank test was used for the median difference between the two groups, and the Kruskal-Wallis test was applied for the median difference between more than two groups.

Multiple linear regressions were performed to explore the associations between childhood abuse with mental distress and PTSD symptoms. We identified confounders for the association-outcome relationships by drawing directed acyclic graphs (DAGs). ${ }^{35}$ We adjusted for confounders but not for mediators since we are interested in an estimation of the total effect of the exposure. If the mediating variables are adjusted for in the model, that path would be closed, and we would no longer see the total effect of the exposure on the outcome. Moreover, it may cause overadjustment bias in the estimation of the total effect and, thereby, reduce precision. ${ }^{36}$ Based on the DAG (figures 1 and 2), age, education and location were identified as confounders in order to estimate the total effect of childhood abuse on the Hopkins Symptom Checklist (HSCL) score (mental distress) and the IES-R score (the PTSD symptoms). We also assessed the interaction between childhood abuse and confounder variables. The level of statistical significance was set at $\mathrm{p} \leq 0.05$ or $95 \%$ CI.

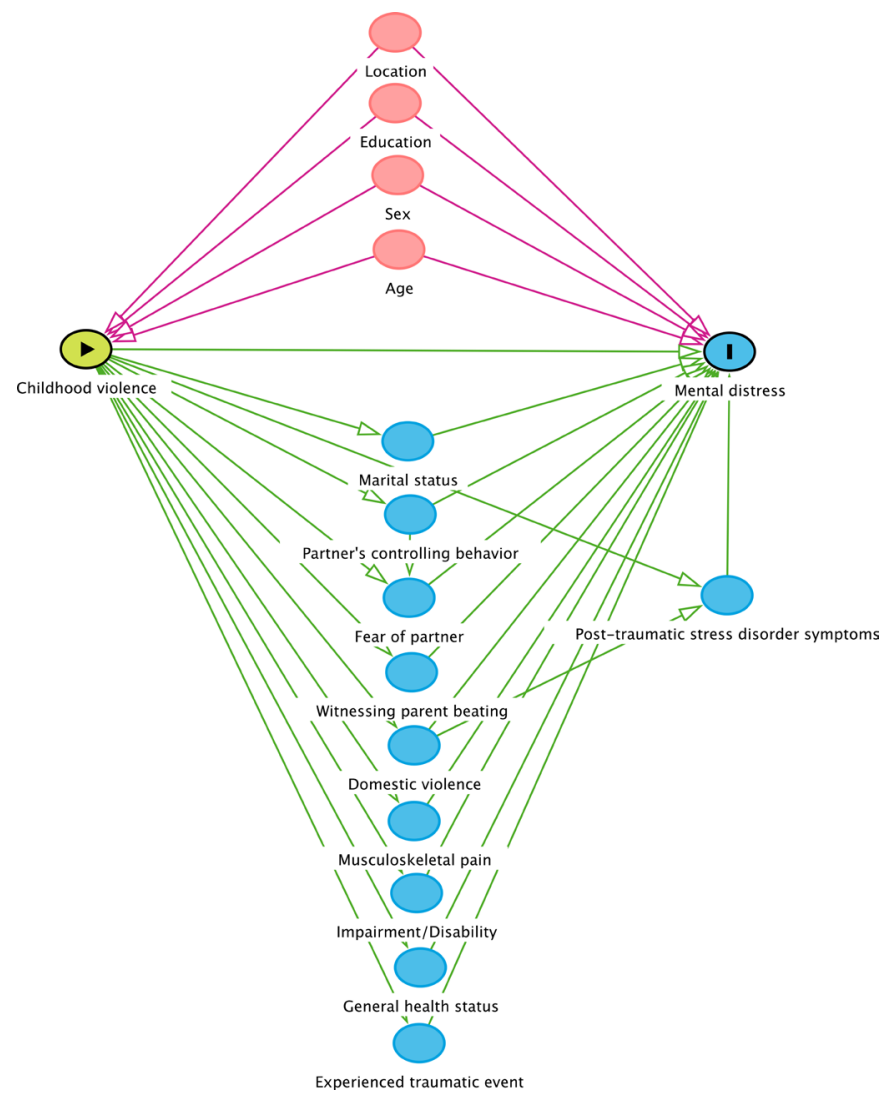

Figure 1 Directed acyclic graph for association between childhood violence and mental distress (Hopkins Symptom Checklist score).

\section{RESULTS}

A total of 2377 participants ( $80.6 \%$ completion rate) were included in the analysis. The sample was evenly distributed between men $(50.1 \%)$ and women $(49.9 \%)$.

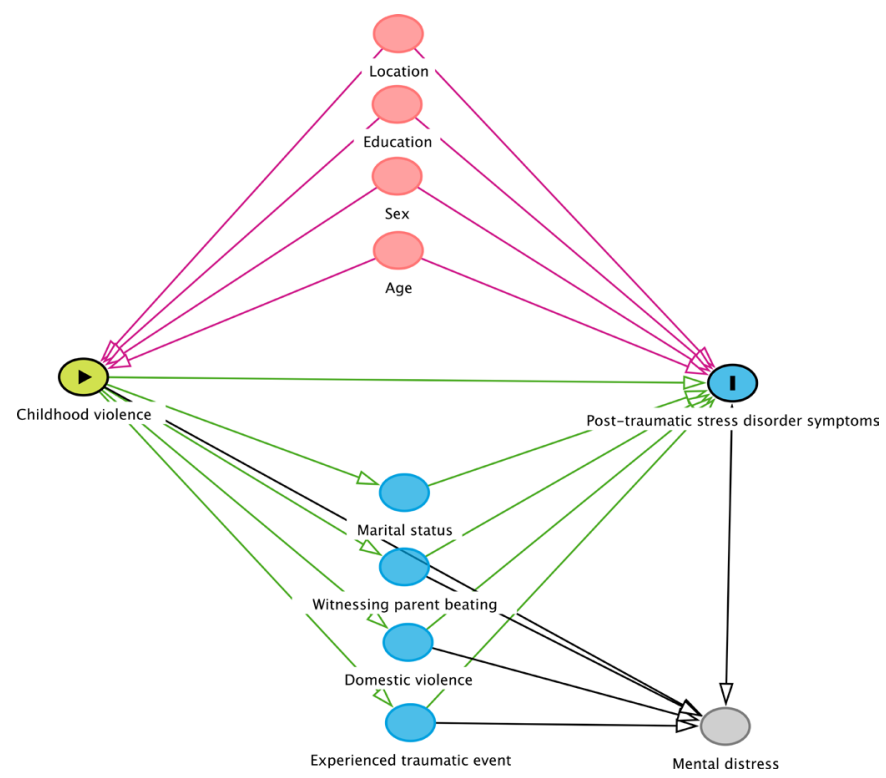

Figure 2 Directed acyclic graph for association between childhood violence and post-traumatic stress disorder symptoms (Impact of Event Scale-Revised). 
Table 1 Prevalence of childhood abuse and PTSD symptoms* among men and women aged 18-49years old in the Yangon Region of Myanmar

\begin{tabular}{|c|c|c|c|c|c|c|c|}
\hline & \multicolumn{2}{|c|}{ Men $(n=1190)$} & \multicolumn{2}{|c|}{ Women $(n=1187)$} & \multirow[b]{2}{*}{$P$ value } & \multicolumn{2}{|c|}{ Total $(\mathrm{N}=2377)$} \\
\hline & N (\%) & $95 \% \mathrm{Cl}$ & N (\%) & $95 \% \mathrm{Cl}$ & & N (\%) & $95 \% \mathrm{Cl}$ \\
\hline Any childhood abuse & $140(12.4)$ & 10.4 to 14.7 & $355(29.8)$ & 25.4 to 34.5 & $<0.001$ & $495(21.1)$ & 18.8 to 23.6 \\
\hline Physical abuse & $79(7.4)$ & 5.7 to 9.5 & 159 (13.3) & 10.2 to 17.2 & 0.003 & $238(10.4)$ & 8.9 to 12.4 \\
\hline Sexual abuse & $19(1.3)$ & 0.8 to 2.2 & 159 (13.3) & 10.2 to 17.2 & $<0.001$ & $178(7.3)$ & 5.7 to 9.3 \\
\hline PTSD symptoms & $62(5.3)$ & 3.7 to 7.6 & $96(7.9)$ & 6.0 to 10.2 & 0.11 & $158(6.6)$ & 5.5 to 7.9 \\
\hline
\end{tabular}

*Impact of Event Scale-Revised score $\geq 20$.

PTSD, post-traumatic stress disorder.

\section{Prevalence of childhood abuse}

The overall prevalence of any childhood abuse (physical, sexual and/or emotional) was $21.1 \%$ (95\% CI $18.8 \%$ to $23.6 \%)$. The prevalence of emotional abuse was $10.4 \%$ (95\% CI $8.8 \%$ to $12.2 \%$ ); that of physical abuse was $10.4 \%$ (95\% CI $8.9 \%$ and $12.4 \%$ ); and that of sexual abuse was $7.3 \%(95 \%$ CI $5.7 \%$ to $9.3 \%$ ) (table 1). Furthermore, the prevalence of childhood abuse reported by women was significantly higher than that reported by men (women: $29.8 \%, 95 \%$ CI $25.4 \%$ to $34.5 \%$ vs men: $12.4 \%$, $95 \%$ CI $10.4 \%$ to $14.7 \%)(\mathrm{p}<0.001)$ (table 1$)$. Likewise, the prevalence of specific types of childhood abuse in women was statistically significantly higher than among men (emotional abuse: $15.2 \%$ women vs $5.6 \%$ men, physical abuse: $13.3 \%$ women vs $7.4 \%$ men and sexual abuse: $13.3 \%$ women vs $1.3 \%$ men) (table 1 ).

\section{Prevalence of traumatic events and PTSDs}

Among the study participants, $560(25.1 \%, 95 \%$ CI $22.4 \%$ to $28.0 \%$ ) reported a traumatic exposure. The prevalence of PTSD symptoms was $6.6 \%$ (95\% CI $5.5 \%$ to $7.9 \%$ ) in the total sample (table 1) and $26.3 \%$ (95\% CI $21.9 \%$ to $31.2 \%$ ) in the trauma-exposed population (data not shown). Although men had a significantly higher prevalence of lifetime traumatic events than women (340 $(31.4 \%)$ vs $220(18.9 \%)) \quad(p<0.001)$, women presented a higher prevalence of PTSD symptoms than men $(41.7 \%$ (95\% CI $35.9 \%$ to $47.6 \%)$ vs $17.0 \%$ (95\% CI $11.9 \%$ to $23.6 \%)(\mathrm{p}<0.001) \quad($ data not shown). Among those reporting traumatic events, $122(22.6 \%, 95 \%$ CI $19.2 \%$ to $26.5 \%$ ) were exposed to childhood abuse: 46 men (36.9\%, $95 \%$ CI $24.9 \%$ to $50.6 \%)$ and 76 women $(63.1 \%$, $95 \%$ CI $49.3 \%$ to $75.0 \%$ ) (data not shown). Of those who were exposed to childhood abuse, $49(37.5 \%, 95 \%$ CI $23.9 \%$ to $53.4 \%$ ) (data not shown) suffered symptoms of PTSD including 8 men (12.0\%) and 41 women (52.4\%).

Bivariate association between childhood abuse and selected sociodemographic, behaviour and health-related factors

Table 2 provides bivariate associations between study variables and childhood abuse by sex. The highest prevalence of childhood abuse was reported by men aged 18-29years old $(15.5 \%)$ and women aged 30-39years old $(39.5 \%)$. Moreover, a significantly higher proportion of childhood abuse was reported by urban than by rural women $(35.6 \%$ vs $24.9 \%, \mathrm{p}=0.03$ ) and by having or having already had a partner compared with never having had a partner in women $(32.5 \%$ vs $20.4 \%, \mathrm{p}=0.04)$. In both men and women, participants who reported their partner's controlling behaviour, being afraid of the partner, witnessing their own parent's beating, and exposure to domestic violence and adult sexual abuse were more likely to have experienced childhood abuse when compared with those who had not had these experiences. We observed no significant bivariate association between childhood abuse with mental distress in either men or women.

\section{Bivariate association between PTSD symptoms and sociodemographic, health-related factors and different types of childhood abuse}

The prevalence of PTSD symptoms by sociodemographic and health-related factors is described in table 3. In both sexes, participants with mental distress presented a significantly higher prevalence of PTSD symptoms than participants without mental distress: $31.9 \%$ vs $13.5 \%$ in men $(\mathrm{p}=0.02)$ and $63.8 \%$ vs $31.7 \%$ in women ( $\mathrm{p}=0.002$ ) (table 3 ). Men who reported witnessing their parents beating and women who experienced emotional abuse in their childhood showed a significantly higher prevalence of PTSD symptoms compared with participants who had lacked of those experiences. The median PTSD symptom score was significantly higher in women exposed to emotional childhood violence (29; IQR: 20) and any childhood violence (23; IQR: 19) as compared with those not exposed to childhood violence

(table 3).

\section{Association between childhood abuse and mental health problems}

In multivariable linear regression, we analysed the association between childhood abuse with mental distress and PTSD symptoms separately for men and women with adjustment for the confounders age, education and location. We found a significant association between childhood abuse and adult mental distress in both men and women (table 4: Model 2). The group exposed to 
Table 2 Prevalence of childhood abuse by sociodemographic, behaviour and other health-related factors among men and women aged 18-49years old in the Yangon Region of Myanmar

\begin{tabular}{|c|c|c|c|c|c|c|}
\hline & \multicolumn{3}{|c|}{ Men $(n=1190)$} & \multicolumn{3}{|c|}{ Women $(n=1187)$} \\
\hline & $\mathbf{N}$ & Prevalence, n (\%) & $P$ value & $\mathbf{N}$ & Prevalence, n (\%) & $P$ value \\
\hline Age group (years) & & & 0.09 & & & $0.02^{*}$ \\
\hline $30-39$ & 406 & $43(9.5)$ & & 393 & $146(39.5)$ & \\
\hline $40-49$ & 344 & $38(11.2)$ & & 342 & $95(29.8)$ & \\
\hline Urban & 566 & $69(13.6)$ & & 568 & $190(35.6)$ & \\
\hline Years of schooling $†$ & & & 0.73 & & & 0.69 \\
\hline$>11$ & 198 & $26(13.0)$ & & 181 & $49(29.4)$ & \\
\hline $6-11$ & 599 & $67(12.9)$ & & 524 & $151(28.5)$ & \\
\hline Having or having already had a partner & 869 & $93(11.0)$ & & 939 & $304(32.5)$ & \\
\hline Mental distress $\ddagger$ & & & 0.56 & & & 0.08 \\
\hline Yes & 168 & $24(14.2)$ & & 271 & $100(36.3)$ & \\
\hline No & 1022 & $116(12.0)$ & & 916 & $255(28.0)$ & \\
\hline General health status & & & 0.79 & & & 0.93 \\
\hline Good & 860 & $96(12.1)$ & & 625 & $189(29.6)$ & \\
\hline Poor & 330 & $44(12.9)$ & & 562 & $166(29.9)$ & \\
\hline Any impairment/disability§ & & & 0.14 & & & 0.43 \\
\hline Yes & 501 & $76(14.8)$ & & 678 & $219(30.9)$ & \\
\hline No & 689 & $64(10.6)$ & & 509 & $136(28.3)$ & \\
\hline Fear of partnerq & & & 0.86 & & & 0.07 \\
\hline Yes & 101 & $12(11.7)$ & & 238 & $90(39.8)$ & \\
\hline No & 768 & $81(10.9)$ & & 699 & $214(30.1)$ & \\
\hline Witnessing parent beating & & & $<0.001^{\star *}$ & & & $<0.001^{* *}$ \\
\hline Yes & 272 & $54(21.4)$ & & 281 & $130(44.4)$ & \\
\hline No & 918 & $86(9.5)$ & & 906 & $225(24.6)$ & \\
\hline Any domestic violence & & & 0.31 & & & $0.003^{*}$ \\
\hline Yes & 445 & $65(13.7)$ & & 560 & $218(38.5)$ & \\
\hline No & 740 & $70(10.9)$ & & 627 & $137(21.3)$ & \\
\hline Adult emotional violence & & & 0.36 & & & $0.02^{*}$ \\
\hline Yes & 137 & $18(9.6)$ & & 251 & $97(39.2)$ & \\
\hline No & 1053 & $122(12.8)$ & & 936 & $258(27.1)$ & \\
\hline Adult physical violence & & & 0.83 & & & 0.07 \\
\hline Yes & 367 & $49(12.7)$ & & 402 & $148(35.5)$ & \\
\hline No & 823 & $91(12.2)$ & & 785 & $207(26.5)$ & \\
\hline Adult sexual violence & & & 0.08 & & & $0.01^{*}$ \\
\hline Yes & 49 & $13(28.4)$ & & 196 & $80(41.0)$ & \\
\hline No & 1136 & $122(11.3)$ & & 991 & $275(27.5)$ & \\
\hline
\end{tabular}




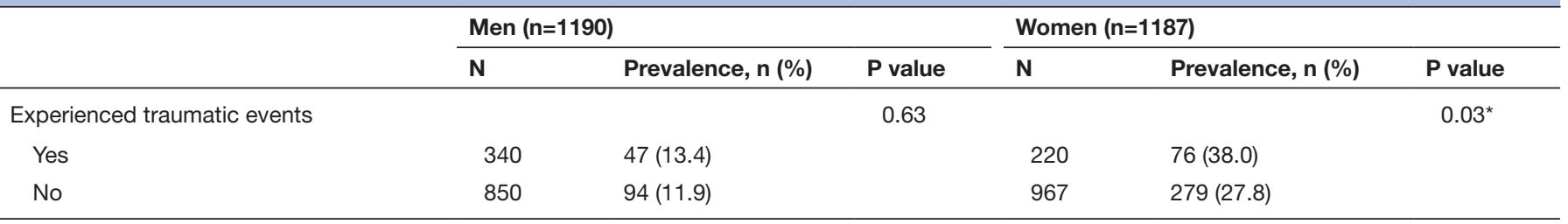

${ }^{* *} \mathrm{P}<0.05,{ }^{* *} \mathrm{P}<0.001$.

†Years at school: $<5$ years (primary education), 6-11 years (secondary education: middle and high school) and $>11$ years (tertiary education: university). ¥Mental distress: $>1.85$ Hopkins Symptom Checklist score.

§Impairment of mobility, hearing, vision, personal hygiene or concentration.

ๆFor married respondents.

childhood abuse showed increased HSCL scores in men ( 0.13 points) and women ( 0.09 points) compared with the group that was not exposed to childhood abuse. However, we detected an interaction between childhood abuse and age in men. Among the younger male respondents, there was no association between exposure to childhood abuse and mental distress when adjusting for the interaction term, whereas the oldest respondents exposed to childhood abuse had a significant 0.33-point increase in HSCL scores compared with those not exposed (table 4: Model 3).

When we examined the association between childhood abuse with PTSD symptoms, childhood abuse was significantly related to adult PTSD symptoms among women (table 4: Model 2). Women exposed to childhood abuse showed a 2.20-point increase in IES-R scores (PTSD symptoms) compared with women not exposed to childhood abuse. Similar to the association between childhood abuse and mental distress, we detected an interaction between childhood abuse and age among men for the association between childhood abuse and PTSD symptoms. No association was found among younger men, but older men exposed to childhood abuse had a significant 2.39-point increase in IES-R scores compared with those who were not exposed (table 4: Model 3). Minor deviations from linearity were detected with the regression models identifying increasing error variances; however, using robust variance estimations did not change the results.

\section{DISCUSSION}

Among 18-49year-old citizens of the Yangon Region of Myanmar, 21\% reported having experienced abuse during childhood. Women reported a significantly higher prevalence than men. Emotional abuse was the type most often reported by women, whereas physical abuse was the most common type reported by men. The prevalence of childhood sexual abuse was significantly higher in women than in men $(13.3 \%$ vs $1.3 \%)$. One-quarter of the study population had experienced traumatic events, and about $26 \%$ of these reported PTSD symptoms, which is approximately $7 \%$ of the total sample population. After adjusting for confounding variables, a significant positive association was found between childhood abuse and adult mental distress and PTSD symptoms in women and in older men.

To the best of our knowledge, the current study is the first population-based study to determine the prevalence of different forms of childhood abuse and to assess the association between childhood abuse and mental health problems in adulthood in Myanmar. The strengths of this study include using personal interviews conducted by well-trained interviewers and a high response rate of $92.6 \%$. However, the analysis was made with $80.6 \%$ of the sample with complete data. The high response rate reduces selection bias, but it also suggests a high level of willingness to participate in a study that addressed sensitive issues about violence. The use of the internationally recommended questionnaires, that is, the NorAQ for childhood abuse, ${ }^{24}$ the IES-R for post-traumatic stress symptoms ${ }^{25}$ and the HSCL-10 for mental distress ${ }^{26}$ further strengthened the study.

The reported prevalence in the various studies is difficult to compare to our study due to differences in methodology, the definition of child abuse applied and the types of abuse investigated, the methods of collecting information, high-risk population study versus population-based study, and the different age groups interviewed. ${ }^{37}$ Some studies focused on a single type of abuse (such as sexual or physical), ${ }^{163839}$ while others examined child maltreatment (physical, sexual, emotional, physical neglect, emotional neglect and witnessing parental violence) in child, adolescent and adult populations. 45891718 38-40

Our findings of any childhood abuse were lower than those of other studies conducted in Norway ${ }^{40}$ and Germany. ${ }^{39}$ The Sami and Norwegian Populations Study 2 (SAMINOR 2) from Norway reported a prevalence of any childhood violence of $25.4 \%{ }^{40}$ in the general population, while in the subpopulation of the Sami minority population, it was high at $36.4 \%$ compared with $22.7 \%$ in the non-Sami population; the latter is similar to our study findings. ${ }^{18}$ When examining the prevalence separately in men and women, our prevalence estimate among women is congruent with the Sami women $(31.1 \%)$, while the prevalence among men in our study is significantly lower than among Sami men $(28.3 \%) .{ }^{18}$ The Sami study used the same type of childhood violence (physical, sexual and emotional violence) and similar measurement tools 
Table 3 Prevalence of post-traumatic stress disorder symptoms $\uparrow$ by sociodemographic and health-related factors among the subgroups who have reported 'experience of traumatic event' from the sample of men and women aged 18-49years old in the Yangon Region of Myanmar

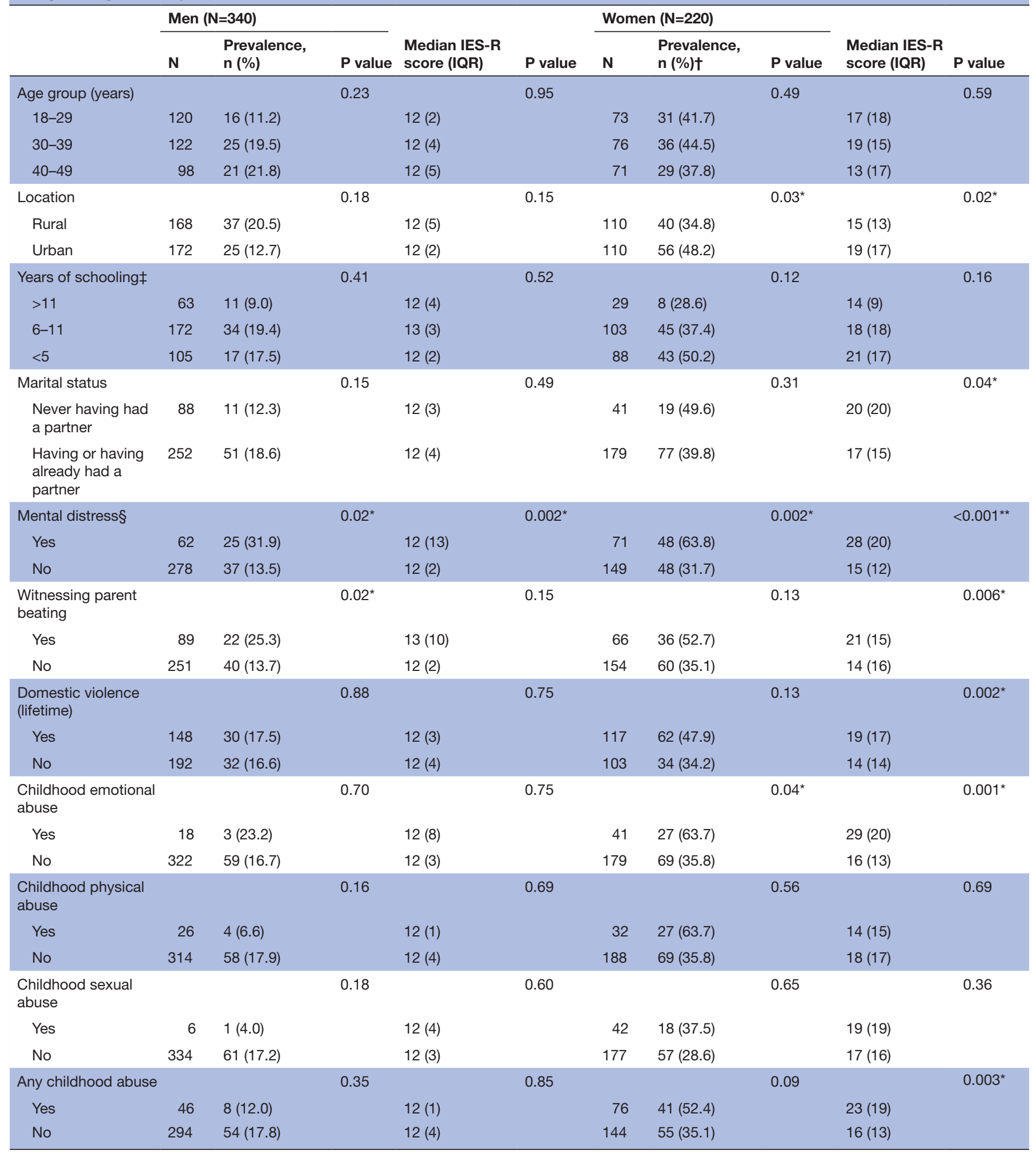

${ }^{*} \mathrm{P}<0.05,{ }^{* *} \mathrm{P}<0.001$

tIES-R score $\geq 20$

†Years at school: $<5$ years (primary education), 6-11 years (secondary education: middle and high school) and $>11$ years (tertiary education: university). \$Mental distress: $>1.85$ Hopkins Symptom Checklist score.

IES-R, Impact of Event Scale-Revised. 
Table 4 Association between any childhood abuse with mental distress (Hopkins Symptom Checklist 10 score) and PTSD symptoms (Impact of Event Scale-Revised score) among men and women aged 18-49years old in the Yangon Region of Myanmar (linear regression analyses)

\begin{tabular}{|c|c|c|c|c|c|c|c|}
\hline & \multirow[b]{2}{*}{ Description } & \multicolumn{3}{|l|}{ Men } & \multicolumn{3}{|l|}{ Women } \\
\hline & & Coefficient & $95 \% \mathrm{Cl}$ & $P$ value & Coefficient & $95 \% \mathrm{Cl}$ & $P$ value \\
\hline \multirow{5}{*}{$\begin{array}{l}\text { (A) Mental } \\
\text { distress }\end{array}$} & Model 1: crude & 0.12 & 0.03 to 0.22 & $0.01^{*}$ & 0.11 & 0.02 to 0.19 & $0.014^{*}$ \\
\hline & Model 2: adjusted $\dagger$ & 0.13 & 0.06 to 0.22 & $0.002^{*}$ & 0.09 & 0.002 to 0.18 & $0.046^{*}$ \\
\hline & Model 3: interaction $\ddagger$ & & & & & & \\
\hline & Age (18years) & -0.01 & -0.17 to 0.16 & 0.92 & & & \\
\hline & Age (49years) & 0.33 & 0.12 to 0.53 & $0.003^{*}$ & & & \\
\hline \multirow{5}{*}{$\begin{array}{l}\text { (B) PTSD } \\
\text { symptoms }\end{array}$} & Model 1: crude & -0.04 & -1.74 to 1.67 & 0.97 & 2.42 & 1.14 to 3.70 & $0.001^{*}$ \\
\hline & Model 2: adjusted $\dagger$ & 0.05 & -1.59 to 1.70 & 0.95 & 2.20 & 0.91 to 3.49 & $0.002^{*}$ \\
\hline & Model 3: interaction $\ddagger$ & & & & - & - & - \\
\hline & Age (18years) & -1.67 & -4.34 to 1.01 & 0.21 & & & \\
\hline & Age (49years) & 2.39 & 0.47 to 4.32 & $0.02^{*}$ & & & \\
\hline
\end{tabular}

${ }^{*} \mathrm{P}<0.05$.

†Adjusted for age, education and location.

$\ddagger$ Adjusted for age, education, location and the interaction term (childhood violencexage).

PTSD, post-traumatic stress disorder.

as the present study. Alternative explanations other than an actual lower prevalence in our study than in the SAMINOR 2 study could be because the SAMINOR 2 study used self-administered questionnaires, which likely allowed participants to feel more comfortable reporting sensitive information and, thereby, reduced the potential for underestimation. Another explanation could be linked to cultural differences. The German populationbased study reported a prevalence of any childhood abuse in the total sample to be $31 \%$ (29.3\% for men and $32.6 \%$ for women) ${ }^{39}$ The German study applied the Childhood Trauma Questionnaire including five types of childhood abuse-physical, emotional and sexual abuse as well as physical and emotional neglect - which may have led to a higher prevalence estimate.

The prevalence of any childhood abuse in our study is substantially higher than that reported by a 2010 population-based Lifestyle and Attitudes towards Sexual Behavior Survey of subjects aged 18-49years old in Japan $(5 \%),{ }^{41}$ which included four types of childhood abuse: physical, sexual and emotional abuse and neglect. By including neglect in the Japanese study, we would have expected a higher prevalence. An explanation for the lower figure could be methodological differences including instrument administration and sampling design. The self-reported surveys may increase the risk of possible false-negative and thus may underestimate the prevalence rates. ${ }^{42}$ Furthermore, the retrospective recall of adverse effects in childhood may cause inaccuracy to estimate the prevalence rates. ${ }^{43}$

For the subgroups of childhood abuse, our results of emotional, physical and sexual abuses during childhood in the total sample are also higher than those of the Japanese study ${ }^{41}$ and the German study, ${ }^{39}$ except for sexual abuse, which was similar to our study. The study from Japan found that, overall, emotional abuse was $4 \%$; physical abuse was $3 \%$; and sexual abuse was $0.6 \% .{ }^{41}$ The German study reported a prevalence of $6.5 \%$ for emotional, $6.7 \%$ for physical and $7.6 \%$ for sexual abuse during childhood. ${ }^{39}$

In contrast, our prevalence estimates of different types of childhood abuse are lower than reported in a national study from Saudi Arabia ${ }^{44}$ and a systematic review study from China. ${ }^{45}$ The Saudi national study applied the selfadministered Adverse Childhood Experiences International Questionnaire, including two items each for emotional and physical abuses and four items for sexual abuse. This study estimated the prevalence of childhood emotional abuse to be $52 \%$, physical $42 \%$ and sexual $21 \%{ }^{44}$ Similarly, the systematic review and meta-analyses from China, which included 62 cross-sectional studies, reported $19.6 \%$ emotional abuse, $26.8 \%$ physical abuse and $8.7 \%$ sexual abuse. ${ }^{45}$ The discrepancies in rates of abuse across studies may reflect cultural values and belief. For example, the behaviours such as corporal punishment are considered abusive in one culture and may be accepted as normal in another culture. The national study from 25 low-income and middle-income countries ${ }^{46}$ found that many cultures traditionally accepted as corporal punishment was normative and necessary to rear children properly. The perception on using corporal punishment ranged from 5\% in Montenegro to 57\% in Sierra Leone. ${ }^{46}$ As in Myanmar's culture, physical abuse including mild to moderate beating (corporal punishment) by a parent, relative or schoolteacher is accepted as normal. It is believed that this punishment will result in children's good behaviour and is disregarded as physical abuse. ${ }^{4}$

In Myanmar, data on child abuse exists resulting from Myanmar's GSHS from $2016^{5}$ and the UNICEF report from 2014 to $2016^{9}$; there are no data among the general 
population. The GSHS was conducted among adolescents aged 13-17years old and investigated physical abuse only $(32.4 \%) .{ }^{5}$ The UNICEF's report stated that $16.2 \%$ and $14.4 \%$ of children experienced physical and sexual abuse, respectively. ${ }^{9}$ The report included 1330 boys and girls aged 9-21 years from the child protection case management services and reported domestic violence, child abuse, neglect, trafficking, exploitation and exposure to armed conflict in 11 townships across the country. ${ }^{9}$ The definition of child abuse used by child protective services is incompatible with our study variables.

Although there is variation in methodological approaches, the rate of exposure to physical, sexual and emotional childhood abuse in the present study is similar to the findings from a recent systematic review of child maltreatment in East Asia and the Pacific Region. ${ }^{47}$ That study reported that the range of different types of abuse included physical abuse $(17 \%-35 \%)$, sexual abuse $(11 \%-$ $22 \%$ ) for girls and $3.0 \%-16.5 \%$ for boys and emotional abuse $18 \%-41.6 \%{ }^{47}$ The review assessed child maltreatment based on a review of publications between 2000 and 2013 in 30 countries in East Asia and the Pacific Region, and both adult and child respondents were included.

In agreement with the study from Japan, ${ }^{41}$ we report that women are more likely to suffer emotional, physical and sexual childhood abuse than men. However, some studies reported that physical abuse is more common among men than women. ${ }^{5} 1848$ A possible explanation may be that boys in some cultures receive harsher treatment from their parents as part of their upbringing. A majority of studies reported that sexual abuse is significantly more prevalent in women than men, which aligns with our study's finding. ${ }^{1841} 44454748$

The prevalence of lifetime traumatic events in our study is in accordance with the German population-based study. ${ }^{49}$ The German study examined eight different types of traumatic events among boys and girls age 14 and older and reported $24 \%$ lifetime traumatic events. ${ }^{49}$ In contrast, the estimate of lifetime traumatic events in a nationally representative 2008/2009 study from Portugal was higher than we reported.$^{50}$ The researchers estimated that $69 \%$ of the population had experienced at least one type of lifetime traumatic event. ${ }^{50} \mathrm{~A}$ longitudinal twin study in England and Wales reported that $31.1 \%$ of the participants had experienced traumatic events by the age of 18 years. ${ }^{51}$ Furthermore, findings from a recent World Mental Health Survey ${ }^{52}$ which assessed 29 different traumatic exposures among the general adult population in 24 countries, reported that the lifetime prevalence of traumatic events varied widely across the countries, ranging from $28.6 \%$ in Bulgaria to $84.6 \%$ in Ukraine. ${ }^{52}$ Variation in the prevalence of traumatic events may partly be explained by the number of traumatic events used across the studies, recall bias and actual differences due to different cultures, levels of violence and conflicts across countries. For example, based on the Global Peace Index in $2014,{ }^{53}$ Bulgaria was ranked as the 28 th most peaceful country in the world, while Ukraine was ranked as 148.
Several studies on the prevalence of PTSD have been conducted in countries within different settings. ${ }^{11-14} 49-51$ The differences in assessment tools, sampling frames, types of traumatic events and other methodological issues limit the comparability of the prevalence rate of PTSD symptoms. The lifetime prevalence of PTSD ranged between $0.3 \%$ in China and $8.8 \%$ in Northern Ireland, which has previously been conflict-ridden. ${ }^{11}$ Our estimated prevalence of PTSD symptoms is within the range of the aforementioned studies. Furthermore, the most recent national representative samples of the general adult population in the $\mathrm{USA}^{54}$ and Portugal ${ }^{50}$ showed that the 12-month prevalence of PTSD was $4.7 \%$ in the USA ${ }^{54}$ and $2.3 \%$ in Portugal, ${ }^{50}$ and lifetime prevalence was $6.1 \%$ in the $\mathrm{USA}^{54}$ and $5.3 \%$ in Portugal. ${ }^{50}$ According to the birth cohort study from England and Wales, ${ }^{51} 7.8 \%$ of young people suffered PTSD by the age of 18 years. Exposure to traumatic events was more prevalent in men than in women; however, the reported rate of PTSD symptoms was higher in women, ${ }^{49-52}$ which supports our findings.

In accordance with international studies, we report that childhood abuse is associated with adult mental health problems, including mental distress and post-traumatic stress symptoms in both men and women. ${ }^{16-18} 55-58 \mathrm{~A}$ recent systematic review of reviews on the consequences of child maltreatment revealed that victims of childhood abuse were more vulnerable to encountering a wide range of negative physical and mental health consequences such as anxiety, depression and PTSDs in adulthood. ${ }^{17}$ A population-based study in Norway, ${ }^{18}$ which applied instruments similar to those used in our study, showed that childhood abuse was significantly associated with adult mental distress and PTSD symptoms in both men and women. In our study, we found that childhood abuse was not significantly associated with mental distress and PTSD symptoms among all men but only older men. A possible explanation might be that older men, compared with younger men, have a higher degree of challenges related to work-related stress, ${ }^{59}$ alcohol consumption, ${ }^{60}$ unhealthy lifestyle habits leading to obesity and hypertension, ${ }^{6162}$ and diseases like cardiovascular disease, diabetes and cancer, which may have led to higher mental distress scores. Moreover, they have experienced more traumatic events because they are older. If there is a link between exposure to childhood abuse and health problems in adulthood and exposure to adulthood violence, the aforementioned may be part of the explanation. Relating to work-related stress, a systematic review study ${ }^{59}$ described that men's work-related stress influences men's mental health problems. A longitudinal study of Korean men ${ }^{60}$ reported that high alcohol consumption among men, compared with normal consumption, was associated with depression. With reference to health problems, a systematic review and meta-analysis study on obesity and depression revealed ${ }^{61}$ that overweight and obesity were risk factors for depression among adults aged 20-59 years, and Scherrer $e t a l^{62}$ reported that cardiovascular disease is a risk factor of depression among middle-aged men. The 
effect of exposure to childhood abuse on mental distress is partly mediated by other factors such as PTSD symptoms,${ }^{55}$ as PTSD occurs concomitant with other psychiatric disorders such as anxiety and depression.

Different forms of childhood abuse may contribute to different mental health problems in adulthood and should be further investigated. Childhood sexual abuse seems to increase the risk of depression and PTSD in adulthood as compared with childhood emotional abuse. ${ }^{567}$

In Myanmar, the child protection and supportive services including physical, psychosocial rehabilitation and counselling services are led by the Ministry of Social Welfare, Relief and Resettlement through the Department of Social Welfare. ${ }^{63}$ However, services and specific policies and programmes for abused children are scarce due to underfunding, lack of well-trained staff for social and counselling services, weak coordination between sectors, and limited outreach to the community. ${ }^{63}$ The effectiveness of a child protection system depends on law and policy. Nevertheless, a revised child law in Myanmar is currently under review. Data on violence against children and its adverse health effects on them throughout their lives are needed, both to highlight the health problem and to inform policies and programmes.

Several limitations need to be taken into consideration when interpreting our findings. We did not invite institutionalised people including soldiers, those hospitalised, and monks and nuns to participate. It is not possible to determine whether these exclusions have led to overestimation or underestimation of our calculations, thereby creating selection bias in the study. It is likely that information bias, especially recall bias and social desirability bias, may have been present. Recall bias may exist as we collected information about violent events experienced in childhood. The abuse-exposed individual might have found it difficult to talk about such a personal experience. Others might have forgotten violent experiences from their childhood, while some respondents may not have wanted to disclose and, thereby, recall the terrible events. Although the interview questions allowed for the clarification of potential misunderstanding better than self-reported questions would have, it is possible that the participants might have been reluctant to report their experiences, especially those related to sexual abuse, because of social desirability bias. ${ }^{43}$ In addition, the majority of abused men might have been more likely to deny their experiences, resulting in a possible underestimation of the prevalence of childhood abuse, particularly among boys. ${ }^{436}$ By contrast, depressed individuals may have a tendency to recall a more negative experience than non-depressed people. ${ }^{64}$ This would potentially have led to an overestimation of the association between childhood abuse and mental health problems. Other limitations include a lack of validation studies on the NorAQ, IES-R (12-item) and HSCL-10 for the studied population. However, these instruments have been validated in other studies and found to be valid. ${ }^{24-33}$ Besides, the 22-item version of IES-R-M has also been validated in the Myanmar population ${ }^{15}$ as well as the validity of the HSCL-10 has been previously addressed. ${ }^{65}$ Furthermore, we back-translated and piloted these instruments in the study population. After a pilot survey, we did key informant interviews with community participants, community leaders and interviewers concerning cultural adaptation of the questionnaires. Then, we discussed the cultural context of the questions with psychologists and minor amendment was done. To summarise the internal validity, we recommend that our findings of prevalence estimates be interpreted with caution as we most likely have underestimated the prevalence of childhood abuse and traumatic events. We lack data on several potential confounders such as parental education and childhood adversity characteristics, ${ }^{66}$ resulting in the possibility of some residual confounding. If it had been possible to include such confounders, it might lead to attenuation of the association between childhood abuse and adult mental health. The cross-sectional design limits the possibility to infer a causal relationship between childhood abuse victimisation with adult mental distress and PTSD symptoms. The generalisation of our main findings might be limited. Data collection was done only in one densely populated region (Yangon); thus, the findings might not apply to other parts of the country, especially not to less densely populated regions.

\section{CONCLUSIONS}

Childhood abuse is prevalent and impacts mental health, leading to problems such as mental distress and PTSD symptoms among adult men and women in the Yangon Region of Myanmar. These findings contribute to a basis for health professionals and policy makers to make evidence-based, coordinated policy and programme changes, including legal and policy reform, improved services for children who have experienced violence and prevention programmes. Interventions aimed at the prevention of childhood abuse such as public awareness campaigns and educational programmes on child abuse could be executed immediately, which would also help Myanmar to achieve the SDG target of 16.2: to end abuse, exploitation, trafficking and all forms of violence and torture against children. We also recommend further nationwide studies on the prevalence and health-related outcomes of violence against children.

\section{Author affiliations}

${ }^{1}$ Community Medicine and Global Health, University of Oslo Faculty of Medicine, Oslo, Norway

${ }^{2}$ Preventive and Social Medicine Department, University of Medicine 2, Yangon, Myanmar

${ }^{3}$ National Norwegian Advisory Board for Concurrent Addiction and Mental Health Problems, Inland Norway University of Applied Sciences, Elverum, Norway ${ }^{4}$ Department of Community Medicine and Global Health, Institute of Health and Society, University of Oslo Faculty of Medicine, Oslo, Norway

Acknowledgements The authors thank all the respondents and local authorities and those from the Yangon Regional Health Department, Department of Public Health, Myanmar, who assisted in the study. The authors also extend their gratitude 
and appreciation to the MY-NORTH project funded by Norad through the NORHED programme.

Contributors WTA contributed to the design of the study, data collection, data analysis and the interpretation and preparation of the draft manuscript. LL participated in the design of the study and commented on the draft. HS conducted data analysis and interpretation of the data. EB was the key contributor for the conception and design of the study and interpretation of the data, and provided intellectual input on the draft. All authors read and approved the final content of the manuscript.WTA act as a gaurantor of this study.

Funding The research was funded by the Norwegian Programme (Norad/NORHED) for Capacity Development in Higher Education and Research for Development through the project entitled Health and Sustainable Development in MyanmarCompetence Building in Public Health and Medical Research and Education, MY-NORTH, Project: MMY-13/0049 (MY-NORTH-1300650).

Competing interests None declared.

Patient and public involvement Patients and/or the public were involved in the design, conduct, reporting or dissemination plans of this research. Refer to the Methods section for further details.

Patient consent for publication Not applicable.

Ethics approval The study was approved for scientific and ethical integrity by the institutional review board, the ethical committee of the University of Public Health, Ministry of Health and Sports in Myanmar (reference number-ethical 5/2016) and the regional committees for Medical and Health Research Ethics (reference number 2016/1195/REK sør-øst A) in Norway. All participants signed a written informed consent form and the interviews took place in complete privacy. All personal IDs were omitted and data were stored to ensure confidentiality.

Provenance and peer review Not commissioned; externally peer reviewed.

Data availability statement Data are available upon reasonable request. Not available for additional data.

Open access This is an open access article distributed in accordance with the Creative Commons Attribution Non Commercial (CC BY-NC 4.0) license, which permits others to distribute, remix, adapt, build upon this work non-commercially, and license their derivative works on different terms, provided the original work is properly cited, appropriate credit is given, any changes made indicated, and the use is non-commercial. See: http://creativecommons.org/licenses/by-nc/4.0/.

ORCID iD

Win Thuzar Aye http://orcid.org/0000-0002-6087-5277

\section{REFERENCES}

1 World Health Organization. Child maltreatment facts sheet, 2016. Available: https://www.who.int/news-room/fact-sheets/detail/childmaltreatment

2 World Health Organization. Global status report on violence prevention, 2014. Available: http://www.who.int/violence_injury_ prevention/violence/status_report/2014/en/

3 Hillis S, Mercy J, Amobi A, et al. Global prevalence of past-year violence against children: a systematic review and minimum estimates. Pediatrics 2016;137:1-13.

4 Fulu E, Miedema S, Roselli T, et al. Pathways between childhood trauma, intimate partner violence, and harsh parenting: findings from the UN Multi-country study on men and violence in Asia and the Pacific. Lancet Glob Health 2017;5:e512-22.

5 World Health Organization and Regional Office for South-East Asia and Ministry of Health and Sports, Myanmar. Report of the second global school-based student health survey (2016) in Myanmar. New Delhi: World Health Organization, 2018.

6 The Global New Light of Myanmar. Stop sexual violence against children, 2018. Available: https://www.globalnewlightofmyanmar. com/stop-sexual-violence-children/

7 Time. A culture of clerical immunity in Myanmar is putting children at risk of abuse, 2018.

8 Unicef for every children, Myanmar. Child protection (facts sheet). Available: https://www.unicef.org/myanmar/child-protection

9 Yarrow CHE, Apland K. Formative evaluation of unicef's strategy and approach to child protection systems building: final report (volume 1). Yangon 2017.

10 Kessler RC, Rose S, Koenen KC, et al. How well can post-traumatic stress disorder be predicted from pre-trauma risk factors? An exploratory study in the WHO world mental health surveys. World Psychiatry 2014;13:265-74

11 Koenen KC, Ratanatharathorn A, Ng L, et al. Posttraumatic stress disorder in the world mental health surveys. Psychol Med 2017;47:2260.

12 Dorrington S, Zavos H, Ball H, et al. Trauma, post-traumatic stress disorder and psychiatric disorders in a middle-income setting: prevalence and comorbidity. Br J Psychiatry 2014;205:383-9.

13 van Wyk S, Schweitzer R, Brough M, et al. A longitudinal study of mental health in refugees from Burma: the impact of therapeutic interventions. Aust N Z J Psychiatry 2012;46:995-1003.

$14 \mathrm{Kim} \mathrm{I}$. Behavioral health symptoms among refugees from Burma: examination of sociodemographic and migration-related factors. Asian Am J Psychol 2018;9:179-89.

15 Htut KM, SS O, Zaw SL. Validation of a Myanmar version of the impact of event Scale-Revised in survivors after disaster 2015.

16 Gallo EAG, Munhoz TN, Loret de Mola C, et al. Gender differences in the effects of childhood maltreatment on adult depression and anxiety: a systematic review and meta-analysis. Child Abuse Negl 2018;79:107-14

17 Carr A, Duff H, Craddock F. A systematic review of reviews of the outcome of Noninstitutional child maltreatment. Trauma Violence Abuse 2020;21:828-43.

18 Eriksen AMA, Hansen KL, Schei B, et al. Childhood violence and mental health among Indigenous SAMI and non-Sami populations in Norway: a SAMINOR 2 questionnaire study. Int $J$ Circumpolar Health 2018;77:1508320.

19 UN General Assembly. Transforming our world: the 2030 agenda for sustainable development. Seventieth session, 2015. Available: https://sustainabledevelopment.un.org/post2015/ transformingourworld

20 Department of Population. Ministry of immigration and population. The 2014 Myanmar population and housing census, the Union report. Nay Pyi Taw: Population Mola, 2015.

21 Ministry of Health and Sports Myanmar. Myanmar demographic and health survey (2015-16). Nay Pyi Taw, Myanmar: Ministry of Health and Sports Myanmar and ICF, 2017.

22 Torgerson DJ, Roberts C. Randomisation methods: concealment. BMJ 1999;319:375-6.

23 World Health Organization. Putting women first: ethical and safety recommendations for research on domestic violence against women in 2001.

24 Swahnberg IMK, Wijma B. The NorVold abuse questionnaire (NorAQ): validation of new measures of emotional, physical, and sexual abuse, and abuse in the health care system among women. Eur J Public Health 2003;13:361-6.

25 Horowitz M, Wilner N, Alvarez W. Impact of event scale: a measure of subjective stress. Psychosom Med 1979;41:209-18.

26 Strand BH, Dalgard OS, Tambs K, et al. Measuring the mental health status of the Norwegian population: a comparison of the instruments SCL-25, SCL-10, SCL-5 and MHI-5 (SF-36). Nord J Psychiatry 2003:57:113-8.

27 Swahnberg K. NorVold abuse questionnaire for men ( $m-N o r A Q)$ : validation of new measures of emotional, physical, and sexual abuse and abuse in health care in male patients. Gend Med 2011;8:69-79.

28 Haddad LG, Shotar A, Younger JB, et al. Screening for domestic violence in Jordan: validation of an Arabic version of a domestic violence against women questionnaire. Int $J$ Womens Health 2011;3:79-86.

29 Weiss DS. The Impact of event scale: revised. In: Wilson JP, CS-k T, eds. Cross-cultural assessment of psychological trauma and PTSD. Boston, MA: Springer US, 2007: 219-38.

30 Gilmoor AR, Adithy A, Regeer B. The cross-cultural validity of posttraumatic stress disorder and post-traumatic stress symptoms in the Indian context: a systematic search and review. Front Psychiatry 2019;10:439.

31 Chen C-S, Cheng C-P, Yen C-F, et al. Validation of the impact of event Scale-Revised for adolescents experiencing the floods and mudslides. Kaohsiung J Med Sci 2011;27:560-5.

32 Thoresen S, Tambs K, Hussain A, et al. Brief measure of posttraumatic stress reactions: impact of event Scale-6. Soc Psychiatry Psychiatr Epidemiol 2010;45:405-12.

33 Syed HR, Zachrisson HD, Dalgard OS, et al. Concordance between Hopkins symptom checklist (HSCL-10) and Pakistan anxiety and depression questionnaire (PADQ), in a rural self-motivated population in Pakistan. BMC Psychiatry 2008;8:59.

34 Lien L, Green K, Thoresen M, et al. Pain complaints as risk factor for mental distress: a three-year follow-up study. Eur Child Adolesc Psychiatry 2011;20:509-16. 
35 Textor J, van der Zander B, Gilthorpe MS, et al. Robust causal inference using directed acyclic graphs: the R package 'dagitty'. Int J Epidemiol 2016;45:1887-94

36 Rothman KJ, Greenland S, Lash TL. Epidemiology: an introduction. Philadelphia: LippincottWilliams \& Wilkins, 2008.

37 Leeb RT, Paulozzi L, Melanson C. Child maltreatment surveillance: uniform Definitions for public health and recommended data elements, version 1.0. Atlanta, GA: Centers for Disease Control and Prevention, National Center for Injury Prevention and Control, 2008.

38 Thoresen S, Myhre M, Wentzel-Larsen T, et al. Violence against children, later victimisation, and mental health: a cross-sectional study of the general Norwegian population. Eur J Psychotraumatol 2015;6:26259.

39 Witt A, Brown RC, Plener PL, et al. Child maltreatment in Germany: prevalence rates in the general population. Child Adolesc Psychiatry Ment Health 2017;11:47.

40 Astrid M. A Eriksen "Breaking the silence" Interpersonal violence and health among Sami and non-Sami. A population-based study in Mid -and Northern Norway. Chapter 5, pg 47.

41 Tsuboi S, Yoshida H, Ae R, et al. Prevalence and demographic distribution of adult survivors of child abuse in Japan 2015;27:NP2578-86.

42 Pereda N, Guilera G, Forns M, et al. The prevalence of child sexual abuse in community and student samples: a meta-analysis. Clin Psychol Rev 2009;29:328-38.

43 Hardt J, Rutter M. Validity of adult retrospective reports of adverse childhood experiences: review of the evidence. J Child Psychol Psychiatry 2004;45:260-73.

44 Almuneef M, ElChoueiry N, Saleheen H, et al. The impact of adverse childhood experiences on social determinants among Saudi adults. Public Health 2018;40:e219-27.

45 Fang X, Fry DA, Ji K, et al. The burden of child maltreatment in China: a systematic review. Bull World Health Organ 2015;93:176.

46 Lansford JE, Deater-Deckard K, Bornstein MH, et al. Attitudes justifying domestic violence predict endorsement of corporal punishment and physical and psychological aggression towards children: a study in 25 low- and middle-income countries. J Pediatr 2014;164:1208-13.

47 United Nations Children's Fund. Violence against children in East Asia and the Pacific: a regional review and synthesis of findings, strengthening child protection series, no. 4, UNICEF EAPRO. Bangkok 2014

48 Chiu GR, Lutfey KE, Litman HJ, et al. Prevalence and overlap of childhood and adult physical, sexual, and emotional abuse: a descriptive analysis of results from the Boston area community health (BACH) survey. Violence Vict 2013;28:381-402.

49 Hauffa R, Rief W, Brähler E, et al. Lifetime traumatic experiences and posttraumatic stress disorder in the German population: results of a representative population survey. J Nerv Ment Dis 2011;199:934-9.

50 Cardoso G, Antunes A, Silva M, et al. Trauma exposure and ptso in Portugal: findings from the world mental health survey initiative. Psychiatry Res 2020;284:112644.
51 Lewis SJ, Arseneault L, Caspi A, et al. The epidemiology of trauma and post-traumatic stress disorder in a representative cohort of young people in England and Wales. Lancet Psychiatry 2019;6:247-56

52 Benjet C, Bromet E, Karam EG, et al. The epidemiology of traumatic event exposure worldwide: results from the world mental health survey Consortium. Psychol Med 2016;46:327-43.

53 Institute for Economics \& Peace. Global peace index 2020: measuring peace in a complex world, Sydney, 2020. http:// visionofhumanity.org/reports

54 Goldstein RB, Smith SM, Chou SP, et al. The epidemiology of DSM-5 posttraumatic stress disorder in the United States: results from the National epidemiologic survey on alcohol and related Conditions-III. Soc Psychiatry Psychiatr Epidemiol 2016;51:1137-48.

55 Klumparendt A, Nelson J, Barenbrügge J, et al. Associations between childhood maltreatment and adult depression: a mediation analysis. BMC Psychiatry 2019;19:36.

56 Friborg $\mathrm{O}$, Emaus $\mathrm{N}$, Rosenvinge $\mathrm{JH}$, et al. Violence affects physical and mental health differently: the general population based Troms $\varnothing$ study. PLoS One 2015;10:e0136588.

57 Jaye Capretto J. Developmental timing of childhood physical and sexual maltreatment predicts adult depression and post-traumatic stress symptoms. J Interpers Violence 2020;35:2558-82.

58 Barboza GE, Dominguez S. Longitudinal growth of post-traumatic stress and depressive symptoms following a child maltreatment allegation: an examination of violence exposure, family risk and placement type. Child Youth Serv Rev 2017;81:368-78.

59 Roche AM, Pidd K, Fischer JA, et al. Men, work, and mental health: a systematic review of depression in Male-dominated industries and occupations. Saf Health Work 2016;7:268-83.

60 Lee SB, Chung S, Lee H, et al. The mutual relationship between men's drinking and depression: a 4-year longitudinal analysis. Alcohol Alcohol 2018;53:597-602.

61 Luppino FS, de Wit LM, Bouvy PF, et al. Overweight, obesity, and depression: a systematic review and meta-analysis of longitudinal studies. Arch Gen Psychiatry 2010;67:220-9.

62 Scherrer JF, Xian H, Bucholz KK, et al. A twin study of depression symptoms, hypertension, and heart disease in middle-aged men. Psychosom Med 2003;65:548-57.

63 UNICEF. Ending violence against children in ASEAN member States: baseline study of priority areas under the ASEAN regional plan of action on the elimination of violence against children. Indonesia 2017.

64 Colman I, Kingsbury M, Garad Y, et al. Consistency in adult reporting of adverse childhood experiences. Psychol Med 2016;46:543-9.

65 Aye WT, Lien L, Stigum H, et al. The prevalence of mental distress and the association with education: a cross-sectional study of 18-49year-old citizens of Yangon region, Myanmar. BMC Public Health 2020;20:94

66 Sheikh MA, Abelsen B, Olsen JA. Clarifying associations between childhood adversity, social support, behavioral factors, and menta health, health, and well-being in adulthood: a population-based study. Front Psychol 2016;7:727. 